Research Article

\title{
Hypoglycemia during Short-Term Intensive Insulin Therapy and Its Association with Long-Term Glycemic Remission in Patients with Newly Diagnosed Type 2 Diabetes
}

\author{
Lijuan Xu $\mathbb{D}^{1},{ }^{1}$ Pengyuan Zhang, ${ }^{1}$ Zhimin Huang, ${ }^{1}$ Liangying Zhong, ${ }^{2}$ Hai Li, ${ }^{1}$ Liehua Liu $\left(\mathbb{D},{ }^{1}\right.$ \\ Juan Liu $(1),{ }^{1}$ Haipeng Xiao $\left(\mathbb{D},{ }^{1}\right.$ and Yanbing $L i \mathbb{1}^{1}$ \\ ${ }^{1}$ Department of Endocrinology, The First Affiliated Hospital, Sun Yat-Sen University, 58th of Zhongshan Er Road, Guangzhou, China \\ 510080 \\ ${ }^{2}$ Department of Laboratory Medicine, The First Affiliated Hospital, Sun Yat-Sen University, 58th of Zhongshan Er Road, Guangzhou, \\ China 510080
}

Correspondence should be addressed to Yanbing Li; liyb@mail.sysu.edu.cn

Received 11 March 2019; Revised 26 January 2020; Accepted 1 February 2020; Published 19 February 2020

Academic Editor: Claudia Cardoso

Copyright (c) 2020 Lijuan Xu et al. This is an open access article distributed under the Creative Commons Attribution License, which permits unrestricted use, distribution, and reproduction in any medium, provided the original work is properly cited.

\begin{abstract}
Background. Short-term intensive insulin therapy induces long-term glycemic remission in half of patients with newly diagnosed type 2 diabetes. The concomitant hypoglycemia needs further analysis. Methods. We collected data from three randomized trials conducted with the same inclusion and exclusion criteria at our institution from 2002 to 2015. Continuous subcutaneous insulin infusion (CSII) was provided to achieve the glycemic goals within a week and then maintained for 14 days. Hypoglycemia episodes during short-term treatment and the one-year drug-free glycemic remission were observed. Results. A total of 244 patients were included. The per day episode of mild hypoglycemia $(3.0-3.9 \mathrm{mmol} / \mathrm{L})$ was higher in the remission group than in the nonremission group $(0.26 \pm 0.20$ vs. $0.18 \pm 0.21, P=0.005)$. However, a moderate hypoglycemia episode $(<3.0 \mathrm{mmol} / \mathrm{L}) \mathrm{per}$ day was insignificantly lower in the remission group $(0.02 \pm 0.04$ vs. $0.03 \pm 0.04, P=0.221)$. After the cessation of insulin treatment, both acute insulin response (491.35 (801.89) vs. 370.22 (542.29), $P=0.028)$ and homeostasis model assessment of insulin resistance $(2.08(2.04)$ vs. $2.48(2.32), P=0.038)$ were more improved in the remission group than in the nonremission group. Logistic regression analysis showed that mild hypoglycemic episodes during short-term CSII treatment were independently related to a long-term glycemic remission $(\mathrm{OR}=2.18,95 \% \mathrm{CI} 1.02 \sim 4.70)$. Stratified analysis demonstrated that episodes during the continuing insulin dose reduction period played a substantial role. Conclusions. Mild hypoglycemic episodes during the continuing insulin dose reduction period indicate a long-term drug-free euglycemic remission in patients with newly diagnosed type 2 diabetes. However, the insulin dosage should be reduced even more quickly in the future treatment to decrease the potential harms.
\end{abstract}

\section{Introduction}

Diabetes is characterized by a progressive deterioration of $\beta$ cell function and reduction in insulin sensitivity $[1,2]$. Glucose toxicity is an undisputed perpetrator of $\beta$-cell dysfunction that not only directly blunts the islet cell response to glucose but also accelerates $\beta$-cell dedifferentiation and apoptosis [3-6]. Short-term continuous subcutaneous insulin infusion (CSII) therapy is reported to rapidly alleviate glucotoxicity and restore the acute insulin response (AIR).
Around half of the patients with newly diagnosed type 2 diabetes responded to CSII therapy and attained a long-term drug-free glycemic remission in our series of studies [7-9]. However, its use to achieve tight glucose control may cause an increase in hypoglycemia [10-12].

Hypoglycemia is classified into three levels based on the standard for hypoglycemia suggested by the recent American Diabetes Association (ADA) guideline [13], i.e., hypoglycemia alert value (mild hypoglycemia), clinically significant hypoglycemia (moderate hypoglycemia), and severe 
hypoglycemia. Severe hypoglycemia and clinically significant hypoglycemia are associated with an increased risk of macrovascular events, microvascular disease, and all-cause death [14-18]. Mild hypoglycemia is traditionally thought to be harmful and should be avoided if possible. However, a growing body of evidence suggests that mild hypoglycemia is not associated with CVD or all-cause mortality [19, 20]. Besides, we noticed that during CSII therapy, after reaching the normal glucose standard, some patients showed repeated mild hypoglycemia episodes despite the fact that their insulin doses decreased sharply. Furthermore, these patients tended to have better long-term drug-free blood glucose control. Whether the onset of mild hypoglycemia marked a longterm glycemic remission was unclear. Whether mild hypoglycemia and moderate hypoglycemia had different influences on long-term glycemic remission was inclusive. In addition to insulin use, what was associated with hypoglycemia was worth discussing.

For the above reasons, we analyzed the features of hypoglycemic episodes during the 2 to 3 weeks of CSII treatment and examined the association of hypoglycemia and the long-term drug-free glycemic control in this study.

\section{Subjects and Methods}

2.1. Subjects. Data was collected from three randomized prospective controlled trials conducted in 2002 2015 with the same inclusion and exclusion criteria (NCT00147836, NCT00948324, and NCT01471808, ClinicalTrials.gov) [8, 9, $21]$. These trials were designed to compare the glucose control of short-term CSII alone with other therapy strategies. Only patients receiving CSII therapy alone were included in the current study to avoid interference from combinations of hypoglycemic agents. All subjects signed informed consent. The study protocols conformed to the provisions of the Declaration of Helsinki and were approved by the local Ethics Committee. Patients, 25 to 70 years old, with newly diagnosed type 2 diabetes, a fasting plasma glucose (FPG) level of $7.0-16.7 \mathrm{mmol} / \mathrm{L}$, and a body mass index (BMI) of $21-35 \mathrm{~kg} / \mathrm{m}^{2}$ were enrolled in these trials. Patients with acute or chronic microvascular complications of diabetes, or who had cardiovascular events or cerebrovascular diseases within 6 months, or who concomitantly suffered from severe diseases, or who had any condition that would greatly influence glycemic levels were excluded. Any other conditions (such as known drug or alcohol abuse or a psychiatric disorder) which might prevent the patient from following the therapeutic advice were also excluded.

2.2. Research Design. As previously described in detail $[8,9$, 21], after the general evaluations and laboratory tests at the baseline, CSII therapy was then provided using insulin Lispro (Humalog, Eli Lilly Inc., USA) or Aspart (NovoRapid, Novo Nordisk, Denmark) to reach the glycemic targets (FPG levels 4.4-6.0 $\mathrm{mmol} / \mathrm{L}$ and $2 \mathrm{~h}$ postprandial glucose (PPG) levels 4.4-8.0 $\mathrm{mmol} / \mathrm{L}$ ) as quickly and steadily as possible. The initial dosage was $0.4-0.8 \mathrm{IU} / \mathrm{kg} /$ day based on the patient's glucose level and physical condition. Half of the dosage was provided as a continuous basal infusion and the other half as boluses divided equally into three premeal infusions. Capillary blood glucose levels were assessed 8 times per day (before and $2 \mathrm{~h}$ after each meal, at bedtime, and at $3 \mathrm{AM}$ ) throughout CSII therapy. Whenever patients felt uncomfortable, the capillary blood glucose was extra checked. After reaching the glycemic targets, CSII therapy was maintained for another 14 days and then the insulin pump was removed before dinner time. The levels of FPG, PPG, HbA1c, and lipid profiles were checked on the next morning after more than 12 hours of discontinuing CSII treatment. Patients were given advice on continuous lifestyle improvements and followed up every 3 months. The one-year drug-free glycemic remission was observed during the follow-up visit. When a hyperglycemic relapse occurred, subsequent therapies were suggested according to the individual's glucose levels.

Glycemic remission was defined as FPG levels $<7.0$ $\mathrm{mmol} / \mathrm{L}$ and $2 \mathrm{~h}$ PPG levels $<10.0 \mathrm{mmol} / \mathrm{L}$ based on daily living measurement and with no need for hypoglycemic agents for at least one year. If the glucose levels exceeded this range, hyperglycemic relapse was considered to have occurred. Patients whose glucose levels met the criteria for glycemic remission were classified as the remission group, and patients whose levels did not meet these criteria were classified as the nonremission group during the one-year follow-up visit [21]. We evaluated the episodes of hypoglycemia during the CSII therapy based on the daily capillary blood glucose levels. A mild hypoglycemic episode was considered when the nadir blood glucose level at the alert value of 3.0-3.9 mmol/L was detected. Moderate hypoglycemia, also called clinically significant hypoglycemia, was regarded as a glucose level $<3.0$ $\mathrm{mmol} / \mathrm{L}$. Severe hypoglycemia was defined as severe cognitive impairment requiring assistance from another person for recovery.

2.3. Measurements. General characteristics and anthropometric indices were evaluated. In particular, daily capillary blood glucose levels and hypoglycemic episodes were recorded. Laboratory indicators, including FPG, $2 \mathrm{~h}$ PPG, glycated hemoglobin (HbAlc), lipid profiles, and high-sensitivity C-reactive protein (hsCRP) levels, were tested. $\beta$-Cell function was estimated by assessing the AIR before and after CSII therapy. Homeostasis model assessment of $\beta$-cell function (HOMA-B) and homeostasis model assessment of insulin resistance (HOMA-IR) were also conducted.

2.4. Statistical Methods. All data were analyzed using the SPSS 22.0 Statistics (IBM, USA). Normally distributed data were expressed as means \pm standard deviations (SDs) and compared by a $t$-test. Nonnormally distributed variables were expressed as medians (interquartile ranges) and analyzed by the rank-sum test. Chi-squared tests were conducted to analyze frequencies. In this study, most variables were normally distributed data. Levels of hsCRP, AIR, HOMA-B, and HOMA-IR were nonnormally distributed variables. The remission rate and the smoking rate were compared by Chi-squared tests. The number of hypoglycemic episodes was evaluated by both $t$-test and rank-sum test. Logistic regression was established to identify the potential influence 
TABLE 1: Differences between the remission group and the nonremission group at the baseline.

\begin{tabular}{|c|c|c|c|c|}
\hline & Remission group & Nonremission group & $t$ or $Z$ value & $P$ value \\
\hline Number & 127 & 117 & - & - \\
\hline Gender (F/M) & $35 / 92$ & $51 / 66$ & 2.613 & $0.011^{*}$ \\
\hline Age (years) & $48.06 \pm 9.85$ & $50.95 \pm 11.70$ & -2.096 & $0.037^{*}$ \\
\hline Smoking habit & $25.20 \%$ & $20.51 \%$ & 0.867 & 0.386 \\
\hline BMI $\left(\mathrm{kg} / \mathrm{m}^{2}\right)$ & $25.53 \pm 3.52$ & $24.66 \pm 3.20$ & 2.007 & $0.046^{*}$ \\
\hline $\begin{array}{l}\mathrm{HbAlc}(\%) \\
\qquad(\mathrm{mmol} / \mathrm{mol})\end{array}$ & $\begin{array}{c}10.54 \pm 2.38 \\
91.00 \pm 14.50\end{array}$ & $\begin{array}{c}10.52 \pm 2.31 \\
91.00 \pm 13.50\end{array}$ & 0.085 & 0.932 \\
\hline FPG (mmol/L) & $12.00 \pm 3.52$ & $12.95 \pm 4.17$ & -1.927 & 0.055 \\
\hline PPG (mmol/L) & $18.52 \pm 6.46$ & $17.34 \pm 5.49$ & 1.503 & 0.134 \\
\hline Triglyceride $(\mathrm{mmol} / \mathrm{L})$ & $2.25 \pm 1.84$ & $2.20 \pm 2.68$ & 0.179 & 0.858 \\
\hline Cholesterol (mmol/L) & $5.72 \pm 1.20$ & $5.93 \pm 1.20$ & -1.371 & 0.172 \\
\hline HDL-C (mmol/L) & $1.09 \pm 0.29$ & $1.17 \pm 0.28$ & -2.147 & $0.033^{*}$ \\
\hline LDL-C (mmol/L) & $3.66 \pm 1.19$ & $3.75 \pm 1.13$ & -0.602 & 0.548 \\
\hline hsCRP (mg/L) & $1.70(2.59)$ & $1.33(2.17)$ & 0.982 & 0.326 \\
\hline AIR (pmol/L·10 min) & $-125.71(263.04)$ & $-99.36(230.29)$ & 0.541 & 0.589 \\
\hline HOMA-B & $24.47(31.99)$ & $23.85(24.02)$ & 1.206 & 0.228 \\
\hline HOMA-IR & $4.20(4.30)$ & $4.88(4.95)$ & 0.893 & 0.373 \\
\hline
\end{tabular}

* means $P<0.05$. BMI: body mass index; HbAlc: glycated hemoglobin; FPG: fasting plasma glucose; PPG: postprandial plasma glucose; HDL-C: high-density lipoprotein cholesterol; LDL-C: low-density lipoprotein cholesterol; hsCRP: high-sensitivity C-reactive protein; AIR: acute insulin response; HOMA-B: homeostasis model assessment of $\beta$-cell function; HOMA-IR: homeostasis model assessment of insulin resistance; AIR: acute insulin response.

of hypoglycemia on glycemic remission. Logistic regression and principal component analyses were used to explore the risk factors of hypoglycemia. A Cox regression model was used to test the association between hypoglycemia and long-term euglycemic remission. The $P$ value $<0.05$ was considered as statistically significant.

\section{Results}

3.1. General Conditions in the Remission Group and the Nonremission Group. We collected data of 124 patients from the clinical trial NCT00147836, 102 patients from NCT00948324, and 33 patients from NCT01471808. These patients received short-term CSII treatment alone. Three patients with slight infection and 12 patients lost to follow-up were excluded. Ultimately, a total of 244 patients with newly diagnosed type 2 diabetes were included in this analysis. After 2-3 weeks of CSII therapy, $127(52.05 \%)$ patients attained one-year glycemic remission.

During the CSII maintaining period, glucose was well controlled within the target range. The levels of FPG were $5.07 \pm 0.48 \mathrm{mmol} / \mathrm{L}$ in the remission group and $5.28 \pm 0.51$ in the nonremission group $(P<0.05)$. Levels of PPG after breakfast, lunch, and dinner were $7.40 \pm 1.24,7.42 \pm 1.18$, and $7.85 \pm 1.39 \mathrm{mmol} / \mathrm{L}$ in the nonremission group and $6.63 \pm 1.05,6.90 \pm 0.97$, and $7.23 \pm 1.19 \mathrm{mmol} / \mathrm{L}$ in the remission group $(P<0.05)$. After $2-3$ weeks of intensive insulin therapy, glucose and lipid profiles were considerably improved. HbAlc was reduced from $10.53 \pm 2.35 \%$ at the baseline to $8.96 \pm 1.83 \%$ after treatment $(P<0.001)$. FPG dropped from $12.48 \pm 3.88 \mathrm{mmol} / \mathrm{L}$ at the baseline to
$6.35 \pm 1.31$ after cessation of CSII overnight $(P<0.001)$. Similarly, PPG significantly decreased from $18.25 \pm$ 6.01 to $8.28 \pm 2.39$ after discontinuing treatment overnight. Comparing the levels at the baseline and after treatment, those of TG, HDL-C, and LDL-C were $2.14 \pm 1.60$ vs. $1.39 \pm 0.59,1.13 \pm 0.28$ vs. $1.23 \pm 0.28$, and $3.71 \pm 1.15$ vs. $3.37 \pm 1.03 \mathrm{mmol} / \mathrm{L}$, respectively $(P<0.001)$. The level of hsCRP was also reduced after treatment compared with that at the baseline (1.30 (2.56) vs. $1.57(2.22), P=0.005)$.

Several differences were identified between the remission and nonremission groups. The proportion of men in the remission and nonremission groups was $58.23 \%$ and $41.78 \%$. And the proportion of women was $40.70 \%$ and $59.30 \%$. Men have a higher glycemic response rate than women $(P=0.011)$. Patients who attained glycemic remission were slightly younger than those who did not ( $48.06 \pm 9.85$ vs. $50.95 \pm 11.70$ years old, $P=0.037$ ). Surprisingly, baseline AIR tended to be lower in the remission group than that in the nonremission group $(-125.71(263.04)$ vs. -99.36 (230.29)), although insignificantly $(P=0.589)$. After short-term CSII treatment, those who attained glycemic remission had more improved AIR (491.35 (801.89) vs. 370.22 (542.29), $P=0.028)$ and HOMA-IR (2.08 (2.04) vs. $2.48(2.32), \quad P=0.038)$ than those who did not (Tables 1 and 2).

3.2. Differences in Insulin Doses and Glucose Profiles between the Remission Group and the Nonremission Group. The initial insulin dose in the remission group was greater than that in the nonremission group $(45.04 \pm 15.45$ vs. $39.44 \pm 12.04 \mathrm{IU}$, $P=0.002$ ), but when body weight was adjusted, the per 
TABLE 2: Differences between the remission group and the nonremission group during and after CSII cessation.

\begin{tabular}{|c|c|c|c|c|}
\hline & Remission group & Nonremission group & $t$ or $Z$ value & $P$ value \\
\hline \multicolumn{5}{|l|}{ After CSII treatment } \\
\hline BMI decrease $\left(\mathrm{kg} / \mathrm{m}^{2}\right)$ & $0.28 \pm 0.80$ & $0.18 \pm 1.02$ & 0.769 & 0.443 \\
\hline $\begin{array}{l}\mathrm{HbA1c}(\%) \\
(\mathrm{mmol} / \mathrm{mol})\end{array}$ & $\begin{array}{c}8.91 \pm 1.86 \\
74.00 \pm 8.50\end{array}$ & $\begin{array}{c}9.03 \pm 1.80 \\
75.00 \pm 8.00\end{array}$ & -0.494 & 0.622 \\
\hline FPG (mmol/L) & $6.07 \pm 1.27$ & $6.66 \pm 1.29$ & -3.626 & $<0.001^{*}$ \\
\hline PPG (mmol/L) & $7.70 \pm 2.28$ & $8.95 \pm 2.43$ & -3.861 & $<0.001^{*}$ \\
\hline Triglyceride (mmol/L) & $1.33 \pm 0.62$ & $1.46 \pm 0.55$ & -1.704 & 0.090 \\
\hline Cholesterol (mmol/L) & $5.24 \pm 1.09$ & $5.37 \pm 1.02$ & -0.943 & 0.347 \\
\hline HDL-C (mmol/L) & $1.22 \pm 0.31$ & $1.25 \pm 0.34$ & -0.639 & 0.524 \\
\hline LDL-C (mmol/L) & $3.40 \pm 1.06$ & $3.34 \pm 0.99$ & 0.436 & 0.663 \\
\hline hsCRP (mg/L) & $1.37(3.01)$ & $1.28(1.71)$ & 0.256 & 0.798 \\
\hline AIR $(\mathrm{pmol} / \mathrm{L} * 10 \mathrm{~min})$ & $491.35(801.89)$ & $370.22(542.29)$ & 2.191 & $0.028^{*}$ \\
\hline HOMA-B & $65.00(73.70)$ & $65.05(66.86)$ & 1.250 & 0.211 \\
\hline HOMA-IR & $2.08(2.04)$ & $2.48(2.32)$ & 2.074 & $0.038^{*}$ \\
\hline \multicolumn{5}{|l|}{ During CSII treatment } \\
\hline Mean insulin dose (IU/kg) & $0.56 \pm 0.17$ & $0.61 \pm 0.16$ & -2.499 & $0.013^{*}$ \\
\hline $\begin{array}{l}\text { Mean insulin dose during } \\
\text { insulin increase stage (IU/kg) }\end{array}$ & $0.74 \pm 0.20$ & $0.73 \pm 0.18$ & 0.423 & 0.673 \\
\hline $\begin{array}{l}\text { Mean insulin dose during } \\
\text { insulin decrease stage (IU/kg) }\end{array}$ & $0.60 \pm 0.23$ & $0.65 \pm 0.22$ & -1.466 & 0.144 \\
\hline \multirow{2}{*}{$\begin{array}{l}\text { Mild hypoglycemic episodes } \\
\text { per day }(3.0 \sim 3.9 \mathrm{mmol} / \mathrm{L})\end{array}$} & $0.26(0.16)$ & $0.11(0.16)$ & 3.518 & $<0.001^{*}$ \\
\hline & $0.26 \pm 0.20$ & $0.18 \pm 0.21$ & 2.824 & $0.005^{*}$ \\
\hline \multirow{2}{*}{$\begin{array}{l}\text { Moderate hypoglycemic episodes } \\
\text { per day }(<3.0 \mathrm{mmol} / \mathrm{L})\end{array}$} & $0(0.05)$ & $0(0.05)$ & 1.097 & 0.273 \\
\hline & $0.02 \pm 0.04$ & $0.03 \pm 0.04$ & -1.228 & 0.221 \\
\hline \multirow{2}{*}{$\begin{array}{l}\text { Mild hypoglycemia episodes per day } \\
\text { during insulin increase stage }\end{array}$} & $0(0.08)$ & $0(0.07)$ & 0.094 & 0.925 \\
\hline & $0.06 \pm 0.12$ & $0.06 \pm 0.13$ & -1.290 & 0.118 \\
\hline \multirow{2}{*}{$\begin{array}{l}\text { Mild hypoglycemia episodes per day } \\
\text { during insulin decrease stage }\end{array}$} & $0.29(0.46)$ & $0.13(0.30)$ & 2.974 & $0.003^{*}$ \\
\hline & $0.34 \pm 0.32$ & $0.22 \pm 0.27$ & 2.820 & $0.006^{*}$ \\
\hline
\end{tabular}

* means $P<0.05$. BMI: body mass index; HbAlc: glycated hemoglobin; FPG: fasting plasma glucose; PPG: postprandial plasma glucose; HDL-C: high-density lipoprotein cholesterol; LDL-C: low-density lipoprotein cholesterol; hsCRP: high-sensitivity C-reactive protein; AIR: acute insulin response; HOMA-B: homeostasis model assessment of $\beta$-cell function; HOMA-IR: homeostasis model assessment of insulin resistance.

kilogram dose became insignificantly higher in the remission group $(0.64 \pm 0.20$ vs. $0.59 \pm 0.16 \mathrm{IU} / \mathrm{kg}, P=0.072)$. There were no differences in insulin dosage peak values between the two groups $(51.35 \pm 18.86$ vs. $47.80 \pm 15.86 \mathrm{IU}, P=$ $0.153 ; 0.72 \pm 0.23$ vs. $0.73 \pm 0.24 \mathrm{IU} / \mathrm{kg}, P=0.964)$. However, the peak-reaching time of the insulin dosage was shorter in the remission group than in the nonremission group $(6.85 \pm 4.37$ vs. $9.07 \pm 4.76$ days, $P<0.001)$. In addition, the insulin dosage before the cessation of CSII was much lower in the remission group than in the nonremission group $(31.88 \pm 20.84$ vs. $41.10 \pm 18.50 \mathrm{IU}, P=0.016 ; 0.45 \pm 0.28$ vs. $0.64 \pm 0.30 \mathrm{IU} / \mathrm{kg}, P<0.001)$. The average daily insulin dose was similar in the two groups during CSII treatment $(40.42 \pm 12.04$ vs. $40.54 \pm 10.72$ IU, $P=0.937)$, but the average dose per kilogram body weight was lower in the remission group than in the nonremission group $(0.56 \pm 0.17$ vs. $0.61 \pm 0.16 \mathrm{IU} / \mathrm{kg}, P=0.013$ ) (Figure 1).

The time of glucose reaching targets was $1.81 \pm 1.04$ days in the remission group and $2.03 \pm 1.04$ days in the nonremis- sion group $(P=0.091)$. Glucose decreased more rapidly and maintained at lower levels during CSII therapy in the remission group, even though the insulin dose was much lower in the remission group than in the nonremission group before CSII therapy stopped. After terminating the short-term CSII therapy overnight, significantly lower FPG and PPG levels were observed in the remission group than in the nonremission group $(6.07 \pm 1.27$ vs. $6.66 \pm$ $1.29 \mathrm{mmol} / \mathrm{L}$ for $\mathrm{FPG} ; 7.70 \pm 2.28$ vs. $8.95 \pm 2.43 \mathrm{mmol} / \mathrm{L}$ for PPG; $P<0.001$ ) (Table 2 and Figure 1 ).

3.3. Hypoglycemic Episodes during CSII Therapy. Although the insulin dose was carefully titrated, patients experienced $0.21 \pm 0.22$ mild hypoglycemia and $0.02 \pm 0.04$ moderate hypoglycemia episodes per day during the $2 \sim 3$ weeks of CSII therapy. Fifteen patients experienced more than one moderate hypoglycemia episode during the $2 \sim 3$ weeks of intensive insulin therapy. No severe hypoglycemia requiring assistance from other individuals occurred; all patients rapidly 


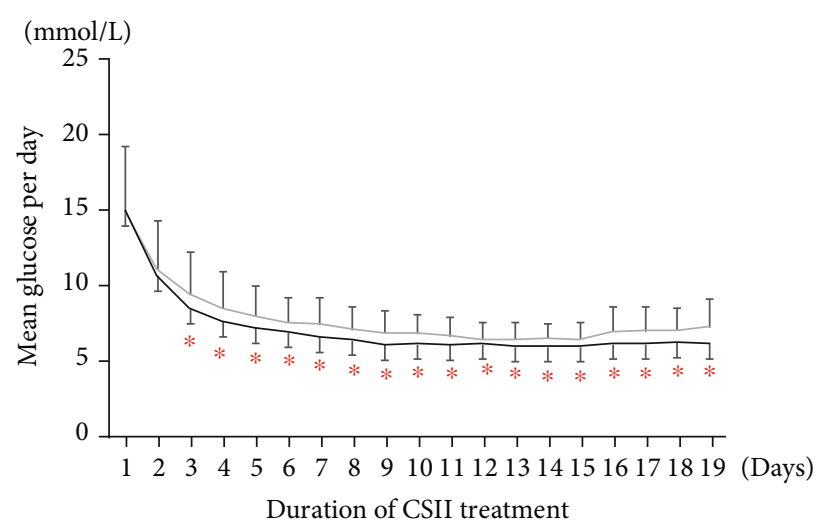

- Nonremission group

— Remission group

(a)

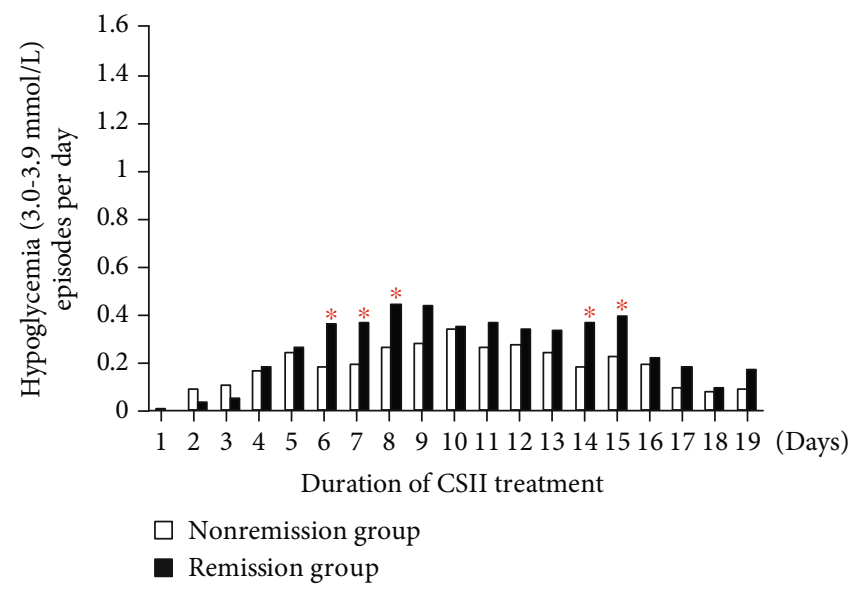

(c)

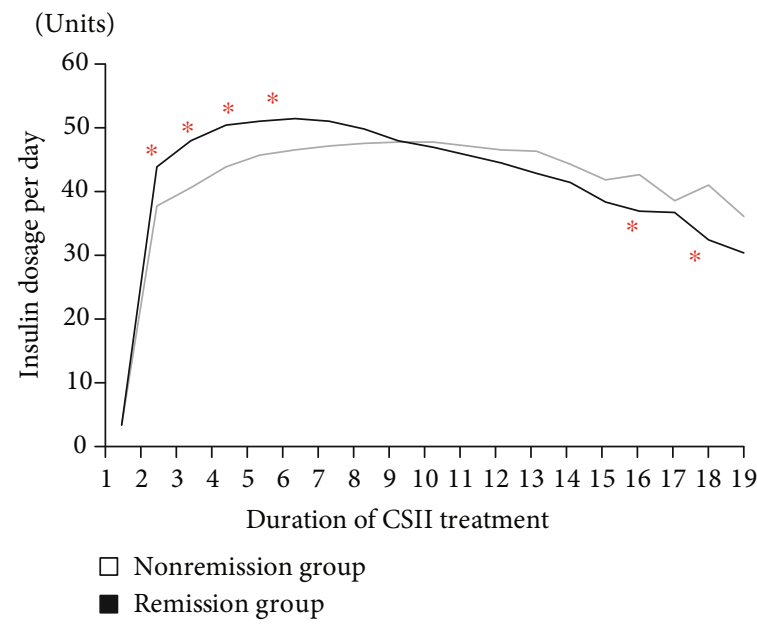

(e)

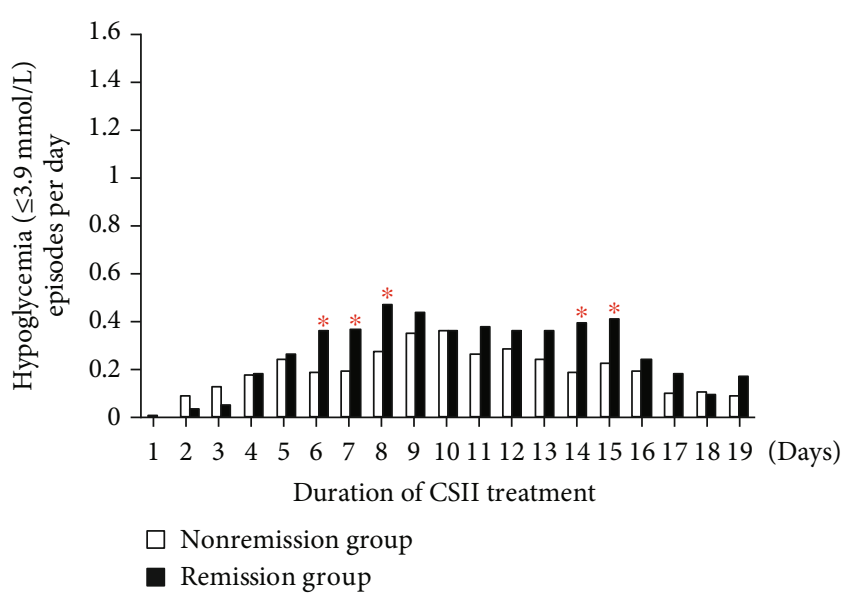

(b)

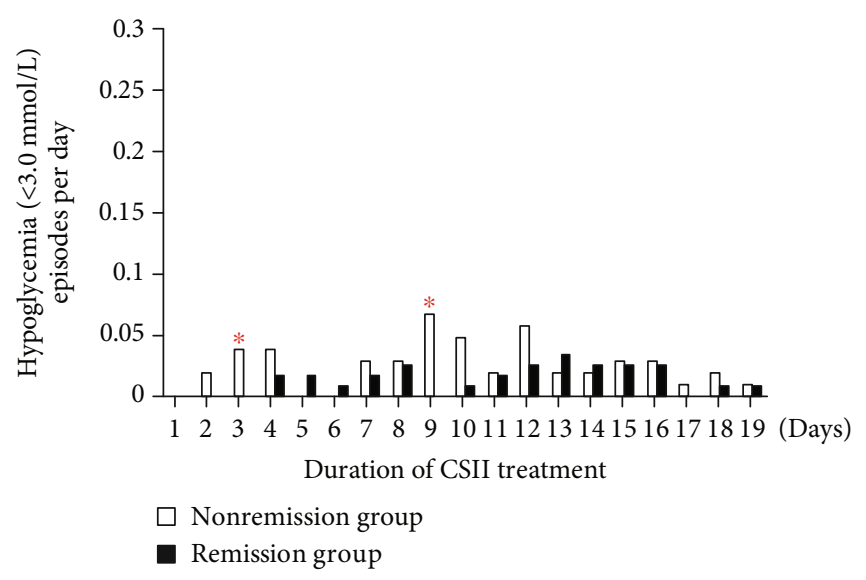

(d)

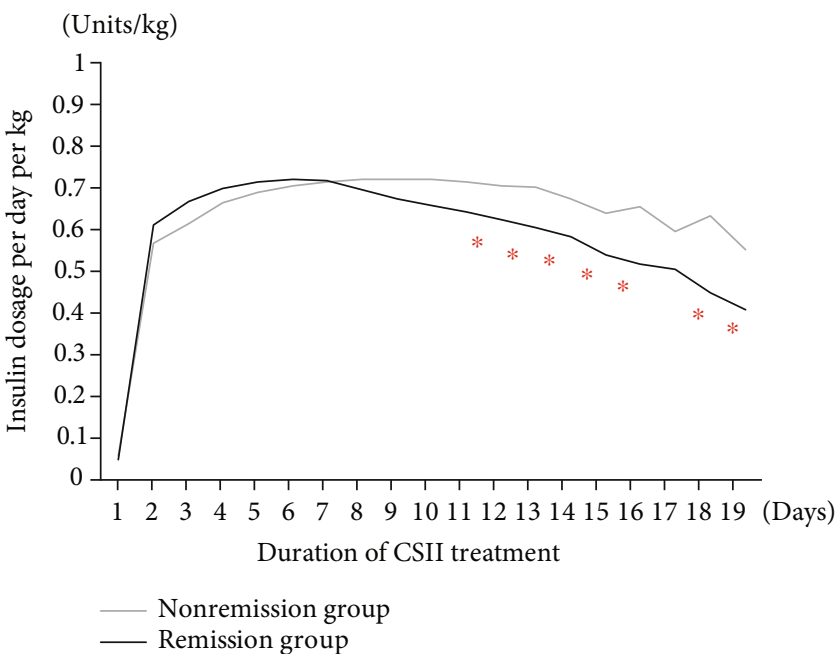

(f)

Figure 1: Glucose profiles (a), daily hypoglycemic episodes (b-d), and daily insulin doses (e, f) of patients during continuous subcutaneous insulin infusion (CSII) therapy in the remission group and the nonremission group. Compared with those in the nonremission group, patients in the remission group had a lower mean glucose, an increased mild hypoglycemia episode, a higher initial insulin dose, and a lower dose before removal of the insulin pump. Glucose profiles (a) and daily insulin doses (e, f) were compared by $t$-test. The number of hypoglycemic episodes (b-d) was calculated by rank-sum tests. $*$ means $P<0.05$. 
recovered following calorie intake and insulin dose titration. No severe adverse events were reported.

Mild hypoglycemia episodes were the most before lunch and the least after supper $(1.18 \pm 1.57$ vs. $0.20 \pm$ $0.52, P<0.001)$. Those in the morning, before bedtime, and at midnight were $0.57 \pm 1.01,0.72 \pm 1.27$, and $0.78 \pm$ 1.38 , respectively $(P=0.100)$. The occurrence of moderate hypoglycemia had the similar trend. The most and least moderate hypoglycemia episodes were $0.11 \pm 0.35$ before lunch and $0.01 \pm 0.11$ after supper $(P<0.001)$. Those in the morning, before bedtime, and at midnight were $0.04 \pm 0.24$, $0.08 \pm 0.36$, and $0.04 \pm 0.21$, respectively $(P=0.698)$.

3.4. The Occurrence of Hypoglycemia in Different Genders and Ages. During CSII treatment, there was no difference between men and women in either mild hypoglycemia $(4.53 \pm 3.98$ vs. $3.76 \pm 3.92, P=0.166)$ or moderate hypoglycemia $(0.40 \pm 0.75$ vs. $0.45 \pm 0.80, P=0.615)$. We divided the patients into the group of $<45$ years old, $45 \sim 55$ years old, and $>55$ years old and observed the occurrence of hypoglycemia in different age groups. Mild hypoglycemia episodes were $4.14 \pm 3.76,4.70 \pm 4.38$, and $4.04 \pm 3.84$ in the three groups during the whole course of CSII treatment $(P=0.794)$. The total episodes of moderate hypoglycemia appeared higher in patients older than 55 years, but not statistically significant $(0.37 \pm 0.74,0.33 \pm 0.60$, and $0.54 \pm 0.89, P=0.153)$.

\subsection{Different Features of Hypoglycemia in the Remission} Group and the Nonremission Group. Significantly more episodes of mild hypoglycemia per day were observed in the remission group than in the nonremission group $(0.26 \pm 0.20$ vs. $0.18 \pm 0.21, P=0.005)$. However, the incidence of moderate hypoglycemia $(<3.0 \mathrm{mmol} / \mathrm{L})$ per day was insignificantly lower in the remission group $(0.02 \pm 0.04$ vs. $0.03 \pm 0.04, P=0.221)$. During the $6-8^{\text {th }}$ day, the episode of mild hypoglycemia in the remission group was significantly higher in the remission group than that in the nonremission group $(0.38 \pm 0.44$ vs. $0.20 \pm 0.36, P=$ $0.001)$. Correspondingly, the mean insulin dose during these days tended to be higher in the remission group $(50.67 \pm 18.96$ vs. $47.17 \pm 14.99 \mathrm{IU} / \mathrm{d}, P=0.154)$. However, on the $14-15^{\text {th }}$ day of insulin therapy, there was a second peak of mild hypoglycemic episodes in the remission group even though the insulin dose was decreased from the maximum to a much lower level $(51.35 \pm 18.86$ vs. $38.28 \pm 20.19 \mathrm{IU} / \mathrm{d}$, $P<0.001$ ), whereas the moderate hypoglycemia episodes did not increase simultaneously. During the whole insulin dose reduction stage, although insulin doses had decreased insignificantly lower in the remission group than in the nonremission group $(37.93 \pm 18.58$ vs. $41.85 \pm 15.87, P=0.122)$, more mild hypoglycemic episodes per day were demonstrated $(0.34 \pm 0.32$ vs. $0.22 \pm 0.27, P=0.006)$. When the rank-sum test of nonnormal distribution was used for the above indices, the same results were obtained (Table 2 and Figure 1).

3.6. Association between Mild Hypoglycemia during the CSII Therapy and the One-Year Euglycemic Remission. Based on the comparisons in the present study, gender, age, BMI, mild hypoglycemic episodes, average insulin dosage of CSII, posttreatment FPG, PPG, AIR levels, and HOMA-IR values significantly differed between the remission and the nonremission groups. Among the above parameters, only mild hypoglycemic episodes ( $\mathrm{OR}=2.18,95 \% \mathrm{CI} 1.02 \sim 4.70)$ and posttreatment FPG levels ( $\mathrm{OR}=0.64,95 \%$ CI $0.46 \sim 0.90$ ) were independently correlated with long-term glycemic remission according to the logistic regression analysis. When these continuous variables were stratified based on the characteristics of clinical indicators, the same association was demonstrated (Table 3). Subgroup analysis showed that mild hypoglycemic episodes during the insulin dosage reduction period instead of increase period were correlated with longterm glycemic remission. Survival curves revealed that patients who experienced only mild hypoglycemia had significantly higher remission rates than patients who either developed moderate hypoglycemia or who experienced no hypoglycemia during the 12-month follow-up $(62.04 \%$ vs. $47.06 \%$ vs. $31.82 \%, P<0.05$, Figure 2 ).

3.7. Baseline Factors Related to Moderate Hypoglycemic Episodes. We compared baseline parameters between patients with moderate hypoglycemia and patients with only mild hypoglycemia and found that those who experienced moderate hypoglycemic episodes had higher baseline levels of HbA1c, FPG, PPG, TC, LDL-C, hsCRP, and initial insulin dosage. According to the principal component analysis and logistic regression analysis, patients with higher glucose levels (HbAlc, FPG, and PPG, OR =2.60, 95\% CI 1.75-3.85, $P<0.001)$ and those with higher cholesterol levels (TC and LDL-C, OR $=1.61,95 \%$ CI 1.14-2.27, $P=$ $0.007)$ at the baseline had an increased risk of moderate hypoglycemia.

\section{Discussion}

In the present study, we analyzed the influence of mild hypoglycemia with nadir glucose levels of 3.0-3.9 mmol/L and moderate hypoglycemia $(<3.0 \mathrm{mmol} / \mathrm{L})$ during the shortterm CSII treatment on the long-term glucose remission. Interestingly, mild hypoglycemic episodes especially during the continuing insulin dose decrease stage were positively correlated with long-term glycemic remission. However, moderate hypoglycemic episodes were insignificantly higher in the nonremission group.

During CSII therapy, there were two peaks of mild hypoglycemia episodes in the remission group. The first peak appeared along with the increase of insulin doses. The second peak occurred on the $15^{\text {th }}$ day of CSII therapy, when insulin doses tended to be lower in the remission group. It suggests a more improved insulin resistance, and thus, the lower dosage of insulin has a larger effect on glucose control and led to hypoglycemia. What is more, the moderate hypoglycemia did not increase simultaneously, indicating a more complete improvement of endogenous islet function. After CSII treatment cessation, both AIR and HOMA-IR demonstrated more significant improvement in the remission group, which also supported our point of view. 
TABLE 3: Influence factors of one-year euglycemia remission.

\begin{tabular}{|c|c|c|c|}
\hline & Odds ratio & $95 \% \mathrm{CI}$ for OR & $P$ value \\
\hline \multicolumn{4}{|l|}{ (A) Overall analysis } \\
\hline Gender & 1.342 & $0.632 \sim 2.850$ & 0.444 \\
\hline Age & 0.992 & $0.961 \sim 1.024$ & 0.616 \\
\hline BMI at baseline & 1.055 & $0.949 \sim 1.172$ & 0.323 \\
\hline HDL-C at baseline & 0.446 & $0.123 \sim 1.613$ & 0.218 \\
\hline Mild hypoglycemia $(3.0 \sim 3.9 \mathrm{mmol} / \mathrm{L})$ & 2.184 & $1.015 \sim 4.699$ & $0.046^{*}$ \\
\hline Mean insulin dose (IU/day) & 0.992 & $0.962 \sim 1.023$ & 0.605 \\
\hline FPG after treatment & 0.641 & $0.456 \sim 0.902$ & $0.011^{*}$ \\
\hline PPG after treatment & 0.912 & $0.787 \sim 1.056$ & 0.217 \\
\hline AIR after treatment & 1.000 & $1.000 \sim 1.001$ & 0.849 \\
\hline HOMA-IR after treatment & 0.976 & $0.807 \sim 1.181$ & 0.806 \\
\hline \multicolumn{4}{|l|}{ (B) Stratified analysis } \\
\hline Gender & 1.439 & $0.673 \sim 3.077$ & 0.348 \\
\hline \multicolumn{4}{|l|}{ Men } \\
\hline \multicolumn{4}{|l|}{ Women } \\
\hline Age (years) & 0.901 & $0.588 \sim 1.380$ & 0.633 \\
\hline \multicolumn{4}{|l|}{$<45$} \\
\hline \multicolumn{4}{|l|}{$45-55$} \\
\hline \multicolumn{4}{|l|}{$>55$} \\
\hline BMI at baseline $\left(\mathrm{kg} / \mathrm{m}^{2}\right)$ & 1.062 & $0.639 \sim 1.767$ & 0.816 \\
\hline \multicolumn{4}{|l|}{$<24$} \\
\hline \multicolumn{4}{|l|}{$24-28$} \\
\hline \multicolumn{4}{|l|}{$>28$} \\
\hline HDL-C at baseline $(\mathrm{mmol} / \mathrm{L})$ & 0.623 & $0.305 \sim 1.269$ & 0.192 \\
\hline \multicolumn{4}{|l|}{$<1.0$} \\
\hline \multicolumn{4}{|l|}{$\geq 1.0$} \\
\hline Total episodes of mild hypoglycemia & 1.593 & $1.153 \sim 2.201$ & $0.005^{*}$ \\
\hline \multicolumn{4}{|l|}{ None } \\
\hline \multicolumn{4}{|l|}{$1-2$} \\
\hline \multicolumn{4}{|l|}{ 3-5 } \\
\hline \multicolumn{4}{|l|}{$>5$} \\
\hline Mean insulin dose (IU/day) & 0.903 & $0.509 \sim 1.600$ & 0.726 \\
\hline \multicolumn{4}{|l|}{$<30$} \\
\hline \multicolumn{4}{|l|}{$30-40$} \\
\hline \multicolumn{4}{|l|}{$>40$} \\
\hline FPG after treatment $(\mathrm{mmol} / \mathrm{L})$ & 0.661 & $0.442 \sim 0.989$ & $0.044^{*}$ \\
\hline \multicolumn{4}{|l|}{$<5.0$} \\
\hline \multicolumn{4}{|l|}{$5.0-6.0$} \\
\hline \multicolumn{4}{|l|}{$6.1-7.0$} \\
\hline \multicolumn{4}{|l|}{$>7.0$} \\
\hline PPG after treatment $(\mathrm{mmol} / \mathrm{L})$ & 0.697 & $0.467 \sim 1.039$ & 0.076 \\
\hline \multicolumn{4}{|l|}{$<6.0$} \\
\hline $6.0-8.0$ & & & \\
\hline $8.1-10.0$ & & & \\
\hline$>10.0$ & & & \\
\hline
\end{tabular}


TABLE 3: Continued.

\begin{tabular}{|c|c|c|c|}
\hline & Odds ratio & $95 \% \mathrm{CI}$ for OR & $P$ value \\
\hline AIR after treatment $(\mathrm{pmol} / \mathrm{L} \cdot 10 \mathrm{~min})$ & 1.333 & $0.718 \sim 2.472$ & 0.362 \\
\hline \multicolumn{4}{|l|}{$<0$} \\
\hline \multicolumn{4}{|l|}{$0-430$} \\
\hline \multicolumn{4}{|l|}{$>430$} \\
\hline HOMA-IR after treatment & 1.271 & $0.666 \sim 2.426$ & 0.466 \\
\hline \multicolumn{4}{|l|}{$<2.25$} \\
\hline$\geq 2.25$ & & & \\
\hline
\end{tabular}

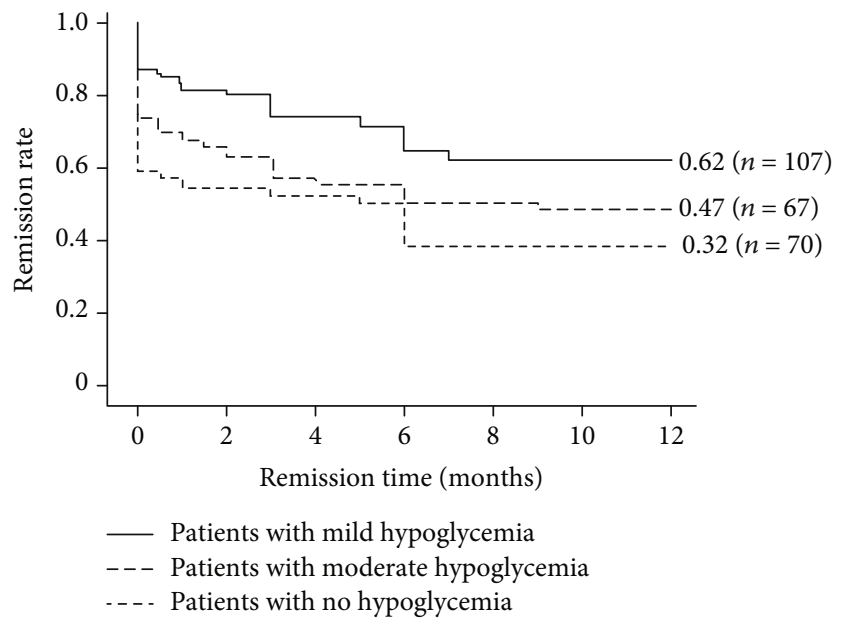

Figure 2: Distinct remission rates during the one-year follow-up in patients with only mild hypoglycemia episode, moderate hypoglycemia episode, or no hypoglycemic occurrence. The Cox regression model was used to test the association between hypoglycemia and long-term euglycemic remission. The remission rate was the highest in patients with mild hypoglycemia $(62.04 \%$ vs. $47.06 \%$ vs. $31.82 \%, P<0.05)$.

This result is partly coincident with another study, in which responders have lower glycemia and less hypoglycemia from the third week onwards of CSII therapy [22], suggesting that 2 weeks of therapy may be needed to improve endogenous islet function when intensive insulin therapy is conducted. However, further studies are necessary to determine the exactly optimal duration of CSII treatment by evaluating the index of $\beta$-cell function and insulin resistance at different treatment courses.

Whereas in our previous study, we did not found differences of hypoglycemia between patients in the remission group and those in the nonremission group [23]. The reason was that in our previous studies, hypoglycemia was not analyzed by subgroups. When hypoglycemia was divided into minor hypoglycemia (3.0-3.9 mmol/L) and moderate hypoglycemia $(<3.0 \mathrm{mmol} / \mathrm{L})$, the results showed a significant difference in mild hypoglycemia between the two groups.

Interestingly, more and more studies showed that mild hypoglycemia was not associated with CVD events and all- cause death $[19,20]$. In the Action in Diabetes and Vascular Disease: Preterax and Diamicron Modified Release Controlled Evaluation (ADVANCE) study, risks of incident macrovascular outcomes and deaths in those experiencing minor hypoglycemia were even found much lower compared to those not reporting minor hypoglycemia [19]. In another study, recurrent mild hypoglycemia had been reported to precondition and protect the brain from neuronal damage and cognitive deficits induced by severe hypoglycemia in rats [24]. Notwithstanding, most studies have shown that mild hypoglycemia might be harmful $[25,26]$. Although in our study mild hypoglycemia episodes during the continuing insulin dose decrease stage were associated with better long-term glucose control, we must caution that the positive correlation between mild hypoglycemia and glycemic remission is only applicable to patients with newly diagnosed type 2 diabetes who do not present with acute or chronic complications or severe concomitant diseases.

Therefore, we need to reduce the insulin dosage even more quickly when blood glucose reaches the target to decrease the occurrence of hypoglycemia as much as possible in the future treatment. Furthermore, moderate hypoglycemic episodes did not contribute to glycemic remission. In addition to the insulin dose, moderate hypoglycemia was associated with baseline glucose levels and lipid profiles. Patients who display higher glucose and cholesterol levels before intensive insulin therapy are at an increased risk of developing moderate hypoglycemia, and their insulin dosage should be adjusted particularly carefully.

In this study, we find that hypoglycemia is more frequent before lunch instead of at midnight. The reasons may be as follows. First, it is related to Chinese breakfast habit. A Chinese breakfast mainly consists of easily absorbed carbohydrates, such as porridge and noodles. These foods cause blood glucose to rise and fall rapidly and thus leading to a decreased level of glucose before the next meal. Second, monitoring midnight glucose only at three o'clock may miss some unrecognized hypoglycemia detection and cause certain bias.

To the best of our knowledge, this study is the first to investigate the impact of mild hypoglycemia (3.0$3.9 \mathrm{mmol} / \mathrm{L})$ and moderate hypoglycemia $(<3.0 \mathrm{mmol} / \mathrm{L})$, respectively, during short-term CSII therapy on longterm glycemic remission. However, several limitations 
should be considered in the interpretation of our results. The current study is a post hoc analysis of three prospective clinical trials. Therefore, we cannot eliminate selection bias and subtle differences among the trials even though these studies were conducted with similar inclusive criteria, exclusive criteria, and insulin dose titration procedure. Another limitation is that although we monitored the glucose levels at $3 \mathrm{am}$, a continuous glucose monitoring system (CGMS) was not provided to most patients, so asymptomatic nocturnal hypoglycemia might have been partly missed in our study. In addition, changes in glycosylated albumin before and after shortterm CSII treatment, which we unfortunately did not check in this study, may better reflect the changes of glucose than $\mathrm{HbAlc}$, considering that glycosylated albumin represents the past 2-3 weeks of glucose.

In conclusion, half of patients with newly diagnosed type 2 diabetes can respond to the short-term intensive insulin treatment and maintain a long-term drug-free glycemic remission. The incidence of mild hypoglycemia is higher, and moderate hypoglycemia tends to be lower in the remission group. Mild hypoglycemia that occurs when the insulin dose continues to decrease indicates a longterm drug-free euglycemic remission. However, in the future treatment with CSII, insulin dosage should be reduced even more quickly when blood glucose reaches the target to decrease the potential harms caused by hypoglycemia.

\section{Data Availability}

The data used to support the findings of this study are available from the corresponding author upon request.

\section{Disclosure}

Parts of this study were presented as a poster at the $78^{\text {th }}$ Scientific Sessions of the American Diabetes Association, Orlando, Florida, 22-26 June 2018.

\section{Conflicts of Interest}

No potential conflicts of interest were reported.

\section{Authors' Contributions}

LJX and PYZ analyzed data and wrote the manuscript. LYZ, LHL, JL, and HL contributed to data collection. LJX, ZMH, HPX, and YBL designed and organized the study and reviewed the manuscript. YBL is the guarantor of this work, who has full access to all data in the study, and takes responsibility for the integrity and accuracy of the data. Lijuan Xu and Pengyuan Zhang contribute equally to this work.

\section{Acknowledgments}

This study was supported by the National Key R\&D Program of China (2018YFC1314100), the Research and Development Program in Key Areas of Guangdong Province
(2019B020230001), the National Natural Science Fund of China (81870557), the National Clinical Key Development Project (Grant 2011), and the 5010 Program of Sun Yat-sen University. We especially thank Dr. Fangfang Zeng, Department of Epidemiology and Biostatistics, Jinan University, for the statistical support.

\section{References}

[1] UK Prospective Diabetes Study Group, "U.K. prospective diabetes study 16 . Overview of 6 years' therapy of type II diabetes: a progressive disease," Diabetes, vol. 44, no. 11, pp. 1249-1258, 1995.

[2] A. E. Butler, J. Janson, S. Bonner-Weir, R. Ritzel, R. A. Rizza, and P. C. Butler, " $\beta$-cell deficit and increased $\beta$-cell apoptosis in humans with type 2 diabetes," Diabetes, vol. 52, no. 1 , pp. 102-110, 2003.

[3] Y. Dor and B. Glaser, " $\beta$-Cell dedifferentiation and type 2 diabetes," The New England Journal of Medicine, vol. 368, no. 6, pp. 572-573, 2013.

[4] C. Talchai, S. Xuan, H. V. Lin, L. Sussel, and D. Accili, "Pancreatic $\beta$ cell dedifferentiation as a mechanism of diabetic $\beta$ cell failure," Cell, vol. 150, no. 6, pp. 1223-1234, 2012.

[5] F. Cinti, R. Bouchi, J. Y. Kim-Muller et al., "Evidence of $\beta$-cell dedifferentiation in human type 2 diabetes," The Journal of Clinical Endocrinology and Metabolism, vol. 101, no. 3, pp. 1044-1054, 2016.

[6] A. Giacca, C. Xiao, A. I. Oprescu, A. C. Carpentier, and G. F. Lewis, "Lipid-induced pancreatic $\beta$-cell dysfunction: focus on in vivo studies," American Journal of PhysiologyEndocrinology and Metabolism, vol. 300, no. 2, pp. E255E262, 2011.

[7] Y. Li, W. Xu, Z. Liao et al., "Induction of long-term glycemic control in newly diagnosed type 2 diabetic patients is associated with improvement of $\beta$-cell function," Diabetes Care, vol. 27, no. 11, pp. 2597-2602, 2004.

[8] J. Weng, Y. Li, W. Xu et al., "Effect of intensive insulin therapy on $\beta$-cell function and glycaemic control in patients with newly diagnosed type 2 diabetes: a multicentre randomised parallel-group trial," The Lancet, vol. 371, no. 9626, pp. 1753-1760, 2008.

[9] Z. Huang, X. Wan, J. Liu et al., "Short-term continuous subcutaneous insulin infusion combined with insulin sensitizers rosiglitazone, metformin, or antioxidant $\alpha$-lipoic acid in patients with newly diagnosed type 2 diabetes mellitus," Diabetes Technology \& Therapeutics, vol. 15, no. 10, pp. 859869, 2013.

[10] E. R. Seaquist, J. Anderson, B. Childs et al., "Hypoglycemia and diabetes: a report of a workgroup of the American Diabetes Association and the Endocrine Society," The Journal of Clinical Endocrinology and Metabolism, vol. 98, no. 5, pp. 18451859, 2013.

[11] M. E. Miller, J. D. Williamson, H. C. Gerstein et al., "Effects of randomization to intensive glucose control on adverse events, cardiovascular disease, and mortality in older versus younger adults in the ACCORD Trial," Diabetes Care, vol. 37, no. 3, pp. 634-643, 2014.

[12] W. Duckworth, C. Abraira, T. Moritz et al., "Glucose control and vascular complications in veterans with type 2 diabetes," The New England Journal of Medicine, vol. 360, no. 2, pp. 129-139, 2009. 
[13] F. Zaccardi, N. N. Dhalwani, D. R. Webb, M. J. Davies, and K. Khunti, "Global burden of hypoglycaemia-related mortality in 109 countries, from 2000 to 2014: an analysis of death certificates," Diabetologia, vol. 61, no. 7, pp. 1592-1602, 2018.

[14] C. Fitzpatrick, S. Chatterjee, S. Seidu et al., "Association of hypoglycaemia and risk of cardiac arrhythmia in patients with diabetes mellitus: a systematic review and meta-analysis," Diabetes, Obesity \& Metabolism, vol. 20, no. 9, pp. 21692178, 2018.

[15] C. J. Huang, W. T. Wang, S. H. Sung et al., "Blood glucose reduction by diabetic drugs with minimal hypoglycaemia risk for cardiovascular outcomes: evidence from meta-regression analysis of randomized controlled trials," Diabetes, Obesity \& Metabolism, vol. 20, no. 9, pp. 2131-2139, 2018.

[16] P. E. Cryer, S. N. Davis, and H. Shamoon, "Hypoglycemia in diabetes," Diabetes Care, vol. 26, no. 6, pp. 1902-1912, 2003.

[17] J. W. Mastaitis, E. Wurmbach, H. Cheng, S. C. Sealfon, and C. V. Mobbs, "Acute induction of gene expression in brain and liver by insulin-induced hypoglycemia," Diabetes, vol. 54, no. 4, pp. 952-958, 2005.

[18] American Diabetes Association, "6. Glycemic Targets:Standards of medical care in diabetes-2018," Diabetes Care, vol. 41, Supplement 1, pp. S55-S64, 2018.

[19] S. Zoungas, A. Patel, J. Chalmers et al., "Severe hypoglycemia and risks of vascular events and death," The New England Journal of Medicine, vol. 363, no. 15, pp. 1410-1418, 2010.

[20] A. O. Y. Luk, T. S. T. Ho, E. S. H. Lau et al., "Association of selfreported recurrent mild hypoglycemia with incident cardiovascular disease and all-cause mortality in patients with type 2 diabetes: prospective analysis of the Joint Asia Diabetes Evaluation Registry," Medicine, vol. 95, no. 45, article e5183, 2016.

[21] L. Xu, L. Wang, X. Huang et al., "Baseline red blood cell distribution width predicts long-term glycemic remission in patients with type 2 diabetes," Diabetes Research and Clinical Practice, vol. 131, pp. 33-41, 2017.

[22] C. M. Stein, C. K. Kramer, B. Zinman, H. Choi, C. Opsteen, and R. Retnakaran, "Clinical predictors and time course of the improvement in $\beta$-cell function with short-term intensive insulin therapy in patients with type 2 diabetes," Diabetic Medicine, vol. 32, no. 5, pp. 645-652, 2015.

[23] L. Liu, J. Liu, L. Xu et al., "Lower mean blood glucose during short-term intensive insulin therapy is associated with longterm glycemic remission in patients with newly diagnosed type 2 diabetes: evidence-based recommendations for standardization," Journal of Diabetes Investigation, vol. 9, no. 4, pp. 908916, 2018.

[24] E. C. Puente, J. Silverstein, A. J. Bree et al., "Recurrent moderate hypoglycemia ameliorates brain damage and cognitive dysfunction induced by severe hypoglycemia," Diabetes, vol. 59, no. 4, pp. 1055-1062, 2010.

[25] J. S. Krinsley, M. J. Schultz, P. E. Spronk et al., "Mild hypoglycemia is independently associated with increased mortality in the critically ill," Critical Care, vol. 15, no. 4, article R173, 2011.

[26] N. Soydan, R. G. Bretzel, B. Fischer, F. Wagenlehner, A. Pilatz, and T. Linn, "Reduced capacity of heart rate regulation in response to mild hypoglycemia induced by glibenclamide and physical exercise in type 2 diabetes," Metabolism, vol. 62, no. 5, pp. 717-724, 2013. 